\title{
STOCHASTICITY AND TIME DELAYS IN GENE EXPRESSION AND EVOLUTIONARY GAME THEORY
}

\author{
Jacek Miękisz \\ University of Warsaw, Institute of Applied Mathematics, Warsaw, Poland
}

Regulation of gene expression is a chemical process involving many coupled elementary chemical reactions modelled usually by systems of differential equations describing time evolution of molecular concentrations. However, due to low numbers of molecules involved in gene expression processes, random fluctuations may play a significant role. To describe stochastic effects in biochemical reactions taking place in living cells, various birth and death processes were studied.

We will review a simple model of protein production which can be completely solved, that is one can obtain analytical expressions for the expected value and the variance of the number of protein molecules [1]. Then we will discuss specific models of mRNA- and protein-regulated networks. In particular, we will discuss contributions of regulatory factors to gene expression noise in four basic mechanisms of negative gene expression control: 1) transcriptional regulation by a protein repressor,

2) translational repression by a protein, 3) transcriptional repression by RNA, and 4), RNA interference with the translation. Our results show that translational repression results in a higher noise than repression on the promoter level [2].

In the standard birth and death processes, products of various reactions appear or degrade immediately after corresponding reactions are triggered. However, many such reactions take a considerable amount of time. Therefore to describe them we have to introduce models with time delays. Reactions with delays are of two kinds: non-consuming and consuming. Reactants of unfinished consuming reactions cannot participate in new reactions, reactants of non-consuming reactions can participate in new reactions. We will discuss simple models of gene expression with time delays. We will analyze both kinetic rate equations and corresponding birth and death processes with both types of time delays. Many kinetic rate equations with non-consuming reactions undergo the Hopf bifurcation when the delay increases and crosses a critical value. For small time delays the system evolves into its stationary state with damped oscillations observed in transient states. We will show that such effects are not present in the case of consuming reactions where for all values of time delay the unique stationary state is asymptotically stable. In the stochastic models corresponding to deterministic rate equations, the variance of the number of protein molecules and autocorrelation functions will be calculated analytically. To deal with more complex models, we will develop a small delay approximation. We compare our results with those obtained earlier in [3].

As we have learned from the above discussion, the effect of time delays on the stability of various dynamical systems depends very much on physical origins of delays. Similar situation appears on a larger time scale in population models. The evolution of populations can be often described within game-theoretic models $[4,5]$. The fundamental notion here is that of an evolutionarily stable strategy. If everybody plays such a strategy, then the small number of mutants playing a different strategy is eliminated from the population. The dynamical interpretation of the evolutionarily stable strategy is provided by a system of differential or difference equations, the so-called replicator equations. They describe the time-evolution of frequencies of strategies. It is known that evolutionarily stable strategies are asymptotically stable stationary points of such dynamics.

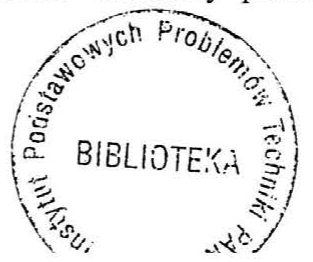


Stochastic stability of evolutionarily stable strategies was analyzed in many papers, see for example $[6,7]$ and a recent review [8].

Various aspects of time delays in replicator dynamics in evolutionary game theory were discussed recently in $[8,9]$. Two specific models of two-player games with two strategies and a unique mixed evolutionarily stable strategy were analyzed. In the social-type model, players imitate opponents taking into account average payoffs of games played some units of time ago. In the biological-type model, new players are born from parents who played in the past. We have shown in [9] that in the first type of dynamics, the unique mixed evolutionarily stable strategy is asymptotically stable for small time delays and becomes unstable for large ones when the population oscillates around its stationary state. In the second type of dynamics, however, the evolutionarily stable strategy is asymptotically stable for any time delay.

The interplay of stochasticity and time delays in evolutionary game theory and other population dynamics models as well as in various models of genetic regulatory networks is a subject of a current intensive research.

Acknowledgments: I would like to thank Polish Ministry of Science and Higher Education for a financial support under the grant N201 023 31/2069.

\section{References}

[1] M. Thattai and A. van Oudenaarden (2001). Intrinsic noise in gene regulatory networks, Proc. Natl. Acad. Sci. USA, 98, 8614-8619.

[2] M. Komorowski, J. Miękisz, and A. M. Kierzek (2009). Translational repression contributes greater noise to gene expression than transcriptional repression, Biophysical Journal, 96, 372-384.

[3] D. Bratsun, D. Volfson, L. S. Tsimring, and J. Hasty (2005). Delay-induced stochastic oscillations in gene regulation, Proc. Natl. Acad. Sci. USA, 102, 14593-14598.

[4] J. Weibull (1995). Evolutionary Game Theory, MIT Press, Cambridge MA.

[5] J. Hofbauer and K. Sigmund (1998). Evolutionary Games and Population Dynamics, Cambridge University Press, Cambridge.

[6] J. Miękisz (2004). Statistical mechanics of spatial evolutionary games, J. Phys. A: Math. Gen. 37, 9891-9906.

[7] J. Miękisz (2005). Equilibrium selection in evolutionary games with random matching of players, J. Theor. Biol. 232, 47-53.

[8] J. Miękisz (2008). Evolutionary game theory and population dynamics, in Multiscale Problems in the Life Sciences, eds. V. Capasso and M. Lachowicz, Lecture Notes in Mathematics, 1940, 269-316.

[9] J. Alboszta and J. Miękisz (2005). Stability of evolutionarily stable strategies 